\title{
artículos
}

\section{La evaluación de la investigación en el contexto de la ciencia}

Martha Añorve Guillén*

\section{RESUMEN}

La au to ra del ar tícu lo pro po ne como pará me tros para evaluar las in ves ti ga ciones, las ca rac te rís ti cas de la cien cia mis ma y sus peculiaridades por áreas. Hace énfasis en las ciencias sociales y en la bibliotecología en particular.

Otros con si de ran dos en la eva lua ción de la in ves ti ga ción tie nen que ver con el con tex to na cio nal ein ternacio nal, la co mu ni dad de la investigación y la administración de la misma; en la medida en que éstas afectan su ejecución.

\begin{abstract}
The author proposes that the characteristics of the self same scien ce as well as its pe cu lia ri ties by areas be used as pa ra me ters in eva lua ting re search. She deals with the so cial scien ces in ge ne ral but with spe cial em phasis on li brary scien ce. Other fac tors to be taken into consideration are the nationalan in ter national con texts, the commu nity where the re search is be ing done and the way in which this is maneged, since these have to do with its being carried out or not.
\end{abstract}

Desde mi punto de vista, la decisión en torno a los criterios para evaluar la investigación estaría determinada en buena medida por la respuesta que se diera a la siguiente pregunta: ¿se con tribu ye con la cien cia al eva luar la in ves ti ga ción? Este plan tea mien to obe de ce al pa pel deci si vo que la in ves ti ga ción juega en el acrecentamiento, cuestionamiento, recreación, etc., de ese creciente cuerpo de ideas y conocimientos al que se denomina ciencia.

La investigación se nos presenta como la forma más prestigiada, en el mundo actual, para arribar (cuestionar, probar, etc.) al conocimiento científico y, a la vez, como un instrumento de la ciencia (implicando en ésta al método).

Des de esta pers pec ti va y de ma nera a priori, parecene ce sa rio re vi sar tam bién el pro ce der de la in ves ti ga ción como ali menta do ra de la cien cia. Como se ha plan tea do al ini cio de esta reflexión, la respuesta está en la ciencia, en lo que de ella se dice, en sus características. Es a partir de éstas y del análisis de lo que es la investigación, don de, des de mi pun to de vis ta, se pue de ana li zar y ha llar la res pues ta a tan de li ca da pero importante cuestión.

De la ciencia en general se dicen cosas tales como que es un conjunto de conocimientos con características de: racionalidad, objetividad, sistematización, exactitud, verificabilidad y, por con si guien te, de fia bi li dad. Uni da a estaidea encontramos que: por medio de la investigación científica el hom bre lle ga a este tipo de co no ci mien to, ya que la in ves ti gación hace posible una reconstrucción conceptual del mundo cada vez más amplia, profunda y exacta.'

“...La investigación científica constituye una intensa actividad conciente, deliberada y racional, la antítesis misma de aquellas nor mas de con duc ta... se mirracionalizadas y se miinconscientes..."(2)

“...La ciencia... es rigurosa, metódica, académica, lógica y práctica. La facilidad...que nos ofrece de comprender sencillamente, de enfocar las cosas con claridad, nos hace sentir que el ins tru men to mis mo es muy real, tan gi ble y de fi ni do., ${ }^{,(3)}$ Esta última parte parece remitirnos al proceder de la investigación y particularmentealmétodo, otro de los puntos considerados para la evaluación.

Entre las mu chas co sas que ca rac te ri zan a la cien cia y su méto do, es tán las men cio na das que, de al gu na manera, propongo como parámetros de evaluación. La evaluación se apunta como demandada por la propia ciencia, en la medida en que busca la verificabilidad, una mayor profundidad y exactitud

* Investigadora del Centro Universitario de Investigaciones Bibliotecológicas de la UNAM 
en el co no ci mien to, al can zable por un proce der y mé to do perfectible y conciente.

Aspectos como los hasta aquí señalados, son aplicables en mayor gra do a las cien cias du ras. Para el caso de las cien cias sociales, el consenso, parámetro de comprobación universal en las lla ma das cien cias, pa re ce no ser del todo apli ca ble, ni a la totalidad de ellas, ni algunos aspectos de las mismas, para ejemplificar revisemos algo de lo apuntado por Siman: "Los críticos literarios y artísticos ocasionalmente pretenden que sus jui cios son tan con vin cen tes que re sul ta ne cio opo ner se a ellos." En es tos ca sos es jus ta men te ne ce sa rio y vá li do el derecho a disentir. En la historia "...los complejos acontecimientos de la historia pocas vecespue den ex plicarsecon vincen te men te en ellen guaje de ele mentales cau sa y efecto..." Se se ña lan cau sas, que si bien son esen cia les, no tie nen la ca racterística de exclusividad que suelen tener en las ciencias duras, por que se bus ca más la dia cro nía de los fe nó me nos, desprendiendo la explicación de sus efectos sincrónicos. Con ello no se quie re de cirque sea ab sur do bus car le yes his tóricas, pues "Acon te cimien tos aparen te men te for tui tos a me nu dosiguen cier tas nor mas y pue den ex pli car se ra cio nal men te." No obstante, señalaelau torcitado “...ningún principiogeneralde explicación histórica ha obtenido — hasta ahora- la aceptación universal..." ${ }^{, 4)}$

En este campo como en la psicología y la filosofía, por mencionar algunos ejemplos, existen grandes escuelas de pensamien to, en oca sio nes an ta góni cas, en don de el con sen so noes el objetivo. No obstante, desde el punto de vista de la autora de este do cu men to, ésto es lo in te re san te a la vez que ca rac terís ti co de es tas dis ci pli nas, y lo que a su jui cio im pul sa la comprensión de los fenómenos.

Todo lo que has ta aquí se ha di cho, nos re mi te a un mun do de la cien cia en ge ne ral fe cun do y com ple jo, don de la com ple jidad y los parámetrostoman otro matiz, en las ciencias sociales, a pe sar de los es fuer zos me to do ló gi cos que en és tas se han hecho, por abordajes más convincentes y fidedignos, que si bien son apli ca bles a al gu nos de sus as pec tos, otros, por su naturaleza, escapan a dichas posibilidades y su forma de argumentación es otra, que aunque aparentemente "menos persuasiva", es igualmente importante en el estudio de los objetos y fenómenos que les son propios.

Entre las disciplinas que se engloban en las denominadas ciencias sociales, se encuentra la bibliotecología, que como ac ti vi dad ha sido ejer ci da des de el mo men to en que el hom bre tuvo ne ce si dad de con ser var y pre ser var da tos y re gis tros. En su carácter de actividad ha sufrido cambios sustanciales con el devenir de los tiempos y de los complejos de información; como el de ir de una mera pre ser va ción y con ser va ción del conocimiento a su puesta en servicio, por señalar alguno; cambios como éste la han mo vi do y ab sor vi do en su ha cer, de jándole poco espacio para la observación, reflexión, análisis, sistematización y teorización de su hacer.

Esta disciplina con relación a otras de su campo, es relativamente nueva en su búsqueda de conocimientocientífico; es a fines del siglo XIX, cuando los cambios ejercidos por las revoluciones europeas se dejan sentir en la bibliotecología en sus aspectostécnicos y de eficiencia. Los aspectos teóricos y su enclave en el proceso de la comunicación se empezaron a dis cu tir en épo cas más re cien tes, a me dia dos del si glo XX. De ahí que para esta jo ven dis ci pli na se pro pon ga, aun, “...la elabo ra ción de sín te sis críti cas... con el ob je to de re va lo rar y replantear los procedimientos de investigación bibliotecológica, so bre todo aque llos orien ta dos a la in ves ti ga ción mis ma... a buscar la expansión del conocimiento... La intención es recapitular información relativa a los componentes básicos de la bibliotecología y sus nexos con la sociodinámica cultural, para ha cer cla ras y for ma les las li gas en tre di ver sos he chos... paraob tenerunaex plicación coherente...y y facilitarelor denamien to de la in ves tiga ción bi blio te co ló gicay la com pren sión de los fenómenos bibliotecarios." ${ }^{(5)}$

Lo que se dice so bre la cien cia en ge ne ral y en par ti cu lar so bre las ciencias sociales, es mucho más de lo aquí mencionado, pudiendo ser aun más complejo que lo señalado. Gran parte del auditorio está consciente de ello. Aunque algunos planteamientos pudieran parecer contradictorios, deben ser revisados y analizados, con miras a sustentar con información y aná li sis el asun to que hoy nos ocu pa, el de la eva lua ción de la investigación.

La ciencia misma nos ofrece esa posibilidad, pues es en sî misma autocorrectiva: "Los modernos sistemas de conocimiento científico son como organismos en crecimiento: mientras están vivos cambian sin pausa... la corrección de erro res es tan va lio sa como el no co me ter los, y ... pro barco sas nue vas e in cier tas es pre fe ri ble a ren dir cul to a las vie jas y ga rantizadas. ${ }^{\prime(6)}$

En la evaluación deben tomarse en cuenta otros elementos como son: los marcosnacionaleseinstitucionales, en los que se desarrollalainvestigación, por su innegableinfluenciasobre ésta, a pesar de que la ciencia como conocimiento no es asunto de propiedad y de frontera. Hay otros elementos relacionados con la personalidad, formas de expresión y de comunicación de los investigadores; de éstos cabe destacar la expresión documen tal de las in ves tiga cio nes, para la que, los estudiosos han aceptado "arbitrajes" en cuanto a su publicación, y valoraciones extrínsecas o intrínsecas de éstos, como fuen te de nue vas in ves ti ga ciones, de avance del co noci miento o de corrección del mismo.

Estas evaluaciones remiten, de alguna manera, a formas de evaluación terminal de la investigación, por árbitros y paráme tros que tie nen que ver con la pu bli ca ción y a la efec tua da también en su fase terminalporinvestigadores. Estefenómeno, por otro lado, parece ser universalmente aceptado por la comunidad dedicada a la investigación. Pero queda aún mucho por analizar y discutir en materia de evaluación de la investigación, ya que es dable enfocarla: como proceso, en su proceso y en la etapa terminal.

Por otra parte, está el considerar quién o quiénes deben evaluar la. En tor no a ésto, es evi den te que no pue den ser otros in- 
dividuos que los versados en materia de investigacióny concretamente en el área de com pe ten cia, pues a pe sar de que en la ciencia hay características comunes, existen diferencias sus tan ciales en los ob je tos de es tu dio y sus for mas de abor darlos.

En la medida en que la mayor parte de la investigaciónse desarrolla actualmente en un marco institucional, la necesidad de es cla re cer el quién y sus fun cio nes re mi te a la ne ce si dad de estudiar los mecanismos y órganos idóneos para efectuar la evaluación, así como el sentido de cada uno de ellos.

Otras pre gun tas fun da men ta les para la eva lua ción de la in vestigación, son el cómo, el por qué y el para qué de la misma, puntos que a pe sar de men cio nar se al fi nal, han sido el mo tor de esta ponencia, sosteniendo, desde el punto de vista de su au to ra, que su en fo que debe es tar en fun ción y al ser vi cio de la ciencia.

Por úl ti mo, hay que ano tar la exis ten cia de otro ele men to contex tual a la in ves ti ga ción y, por tan to, de im por tan cia al to car el pro ble ma de la eva lua ción de la mis ma, éste es el que re mi te a la administración de la investigación cuyas características preconforman de alguna manera a la misma.

\section{RECOMENDACIONES}

Evaluar lain ves ti ga ción con for me a las carac terís ti cas y parámetros de la ciencia y sus peculiaridades por áreas.

En la evaluación deben tomarse en cuenta también el marco nacionalein ternacional, la personalidad, las for mas de ex presión y co mu ni ca ción pro pias de la co mu ni dad que se de di ca a la in ves ti ga ción; el quién o quié nes de ben eva luar la, el cómo, el por qué y el para qué; las características contextuales de la investigación, como es la administración de la misma.

Respecto del quién debe evaluar, se resalta la necesidad de que sean personas versadas en la investigación y concretamente en el área o temática específicas.

\section{CITAS BIBLIOGRAFICAS}

1 Bunge, Mario. La ciencia, su método y su filosofía. Buenos Aires: Siglo Veinte, 1975. p. 9

2 Zi man, John. El conocimien to público: unen sa yo sobrela dimen sión socialdelacien cia. Mé xico : Fondo de Cultura Económica, 1972. p. 11

3 Ziman, Op. cit., p. 14

$4 \quad$ ibid p. $32,34-35$

5 La fuente, Ramiro. "Lasínte sis crítica del conoci mien to biblio te co ló gi co: su valor para la in ves tiga ción biblio te co ló gi ca.”p.3. En:InvestigaciónBibliotecológica:archivonomía,bibliotecología e información. vol. 2, no. 4 (enero-junio, 1988)

$6 \quad$ Bunge, Op. cit., p. $33-34$

\section{OBRAS CONSULTADAS}

Bunge, Mario. La ciencia, su método y su filosofía. Buenos Aires : Siglo Veinte, 1975. 110 p.

Lafuen te, Ramiro. "La sín te sis crítica del conoci mien to biblio te coló gico: su valor para la in ves tigación bibliote cológica.”p.3-10.En InvestigaciónBibliotecológica:archivonomía,bibliotecología e información. vol. 2, no. 4. (enero-junio, 1988).

Zi man, John. El conocimien to público: unen sayo sobrela dimen sión socialdelaciencia.México : Fondo de Cultura Económica. 1972. 189 p. 